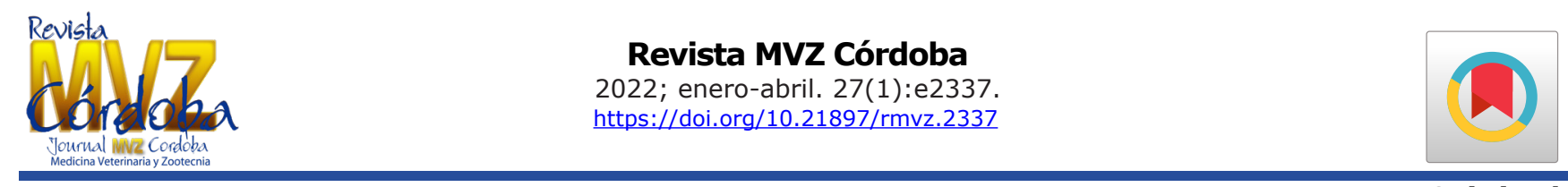

Original

\title{
Bioacumulación de mercurio y plomo en el pato Dendrocygna autumnalis en la subregión de la Mojana, Colombia
}

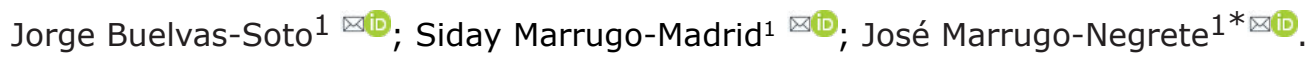

\footnotetext{
${ }^{1}$ Universidad de Córdoba, Laboratorio de Toxicología y Gestión Ambiental, Grupo de Química del Agua, Aplicada y Ambiental, Montería, Colombia.

*Correspondencia: jmarrugo@correo.unicordoba.edu.co
}

Recibido: Abril 2021; Aceptado: Octubre 2021; Publicado: Diciembre 2021.

\section{RESUMEN}

Objetivo. La Mojana es una zona biodiversa de humedales que se ubica en la región Caribe, siendo el hábitat de numerosas especies. La minería, aguas arriba, y las actividades agrícolas han generado la acumulación de metales pesados en estos ecosistemas. Se determinaron las concentraciones de mercurio $(\mathrm{Hg})$ y plomo $(\mathrm{Pb})$ en 47 muestras de sangre y plumas de Dendrocygna autumnalis (Pisingo) en dos sitios de La Mojana, Colombia. Materiales y métodos. Las concentraciones de $\mathrm{Hg}$ y $\mathrm{Pb}$ en sangre y plumas se cuantificaron mediante espectrofotometría de absorción atómica de vapor frío (CVAAS) y espectrometría de absorción atómica en horno de grafito (GFAAS), respectivamente. Resultados. Las más altas concentraciones de $\mathrm{Hg}$ y $\mathrm{Pb}$ en plumas y sangre fueron $475.4 \pm 180.5$ $\mathrm{ng} / \mathrm{g}, 873.59 \pm 412.06 \mathrm{ng} / \mathrm{g}$ y $9.87 \pm 10.6 \mathrm{ng} / \mathrm{mL}, 218.5 \pm 216.6 \mathrm{ng} / \mathrm{mL}$, respectivamente. El $100 \%$ de las muestras de plumas de los individuos capturados sobrepasó el límite permisible de $40 \mathrm{ng} / \mathrm{g}$ para $\mathrm{Hg}$ en carne de pato establecido por la Unión Europea. Así mismo, el $47 \%$ de las muestras de sangre y el $53 \%$ de las de plumas de los individuos capturados excedieron los límites permisibles para plomo en carne de pato de $100 \mathrm{ng} / \mathrm{g}$ de la Unión Europea. Conclusiones. Las actividades mineras y agrícolas han generado procesos de acumulación de metales pesados en Dendrocygna autumnalis, lo cual representa una amenaza para la conservación de esta especie y un riesgo a la salud por consumo para los habitantes de la región.

Palabras clave: Aves; bioindicadores; humedales; metales pesados; minería; toxicología ambiental (Fuentes: Tesauro ICYT de Biología Animal, Tesauro ambiental para Colombia).

\section{ABSTRACT}

Objective. La Mojana is a biodiverse area of wetlands that is in the Caribbean region, being the habitat of numerous species. Mining, upstream, and agricultural activities have generated the accumulation of heavy metals in these ecosystems. Mercury $(\mathrm{Hg})$ and Lead $(\mathrm{Pb})$ concentrations in 47 samples of blood and feathers of Dendrocygna autumnalis (Pisingo) were determined in two sites at the Mojana, Colombia. Materials and methods. Concentrations of $\mathrm{Hg}$ and $\mathrm{Pb}$ in blood and feathers were quantified using Cold Vapor-Atomic Absorption Spectrophotometry (CVAAS) and graphite furnace atomic absorption spectrometry (GFAAS), respectively. Results. The highest concentrations of $\mathrm{Hg}$

Como citar (Vancouver).

Buelvas-Soto J, Marrugo-Madrid S, Marrugo-Negrete J. Bioacumulación de mercurio y plomo en el pato Dendrocygna autumnalis en la subregión de la Mojana, Colombia. Rev MVZ Córdoba. 2022; 27(1):e2337. https://doi.org/10.21897/rmvz.2337

CEl (los) autor (es) 2021. Este artículo se distribuye bajo los términos de la licencia internacional Creative Commons Attribution 4.0 (https:// cc) $\$$ creativecommons.org/licenses/by-nc-sa/4.0/), que permite a otros distribuir, remezclar, retocar, y crear a partir de su obra de modo no comercial, BY NC SA siempre y cuando den crédito y licencien sus nuevas creaciones bajo las mismas condiciones. 
and $\mathrm{Pb}$ in feathers and blood were $475.4 \pm 180.5 \mathrm{ng} / \mathrm{g}, 873.59 \pm 412.06 \mathrm{ng} / \mathrm{g}$ and $9.87 \pm 10.6 \mathrm{ng} /$ $\mathrm{mL}, 218.5 \pm 216.6 \mathrm{ng} / \mathrm{mL}$, respectively. $100 \%$ of the feather samples from the captured individuals exceeded the permissible limit of $40 \mathrm{ng} / \mathrm{g}$ for $\mathrm{Hg}$ in duck meat established by the European Union. Likewise, $47 \%$ of the blood samples and $53 \%$ of the feathers from the captured individuals exceeded the European Union permissible limits for lead in duck meat of $100 \mathrm{ng} / \mathrm{g}$. Conclusions. Mining and agricultural activities have generated heavy metal accumulation processes in Dendrocygna autumnalis, which represents a threat to the conservation of this species and a risk to the health of the inhabitants of the region due to its consumption.

Keywords: Birds; bioindicators; wetlands; heavy metals; mining; Environmental toxicology (Sources: ICYT Animal Biology Thesaurus, Environmental Thesaurus for Colombia).

\section{INTRODUCCIÓN}

La ecorregión de La Mojana representa uno de los sistemas de humedales más importantes del Caribe Colombiano, la cual se origina por la interacción de las cuencas de tres ríos: Cauca, Magdalena y San Jorge (1). Estos humedales ocupan 30.781.149 hectáreas del país (2), de los cuales, 760.340 hectáreas están reconocidos como territorios RAMSAR (3), ecosistemas con una vasta biodiversidad que son fundamentales en los ciclos ecológicos, además de brindar servicios ecosistémicos a las poblaciones humanas asociadas a ellos (4).

La biodiversidad y ecosistemas de La Mojana han sido afectados por la minería aurífera, la cual descarga metales pesados en los ríos San Jorge, Cauca y Magdalena. Dichos ríos circundan los humedales de la región de La Mojana y transportan los metales pesados hasta esta (1).

Un gran número de estudios han registrado altos niveles de contaminación por metales pesados como $\mathrm{As}, \mathrm{Cr}, \mathrm{Cd}, \mathrm{Hg}, \mathrm{Pb}, \mathrm{Ni}$, en diversos componentes ecosistémicos de La Mojana. Se ha registrado concentraciones de metales pesados en sedimentos y materia orgánica tales como $\mathrm{Cu}$ (48400 ng/g), Zn (79200 ng/g), Ni (58100 ng/g), $\mathrm{Pb}$ (3200 ng/g), Cd (560 ng/g), Mn (41100 ng/g), y Hg $(100 \mathrm{ng} / \mathrm{g})(5)$, concentraciones de $\mathrm{HgT}$ de $0.097 \pm 0.049 \mu \mathrm{g} / \mathrm{g}$ en sedimentos, $0.191 \pm 0.017$ $\mu \mathrm{g} / \mathrm{g}$ en macrófitas (1), $17.05 \mathrm{ng} / \mathrm{g}$ en arroz (6), en peces como Caquetaia kraussi (150 \pm 57 $\mathrm{ng} / \mathrm{g})$, Hoplias malabaricus (239 $\pm 67 \mathrm{ng} / \mathrm{g})$, Plagioscion surinamensis $(578 \pm 392 \mathrm{ng} / \mathrm{g})$ y Pseudoplatystoma fasciatum (568 $\pm 224 \mathrm{ng} / \mathrm{g}$ ) (1).

Dicha contaminación tiene su origen principalmente en la minería del oro y en los insumos agroquímicos utilizados en los cultivos de interés comercial (1). De los metales pesados que generan procesos de contaminación en
La Mojana revisten gran importancia por su abundancia, distribución y efectos en la biota el mercurio $(\mathrm{Hg})$ y el plomo $(\mathrm{Pb})(5)$. El $\mathrm{Hg}$ origina problemas a nivel reproductivo, embriológico, etológico, nervioso, inmunológico y genético en el desarrollo de las aves (7). Por su parte, la contaminación con $\mathrm{Pb}$ en las aves genera problemas neuromotores, anemia, letargo, disminución de la masa corporal, alteraciones comportamentales, reproductivas, además de afectar el desarrollo embrionario (8).

Del total de especies de aves que posee Colombia, 266 especies son aves asociadas a ecosistemas acuáticos (9), las cuales tienen una gran importancia como bioindicadores de los estados de conservación de los humedales y en los procesos ecológicos que se presentan en estos ecosistemas (10). La Mojana, de manera permanente y temporal, alberga a un gran número de especies de aves que se distribuyen entre los diferentes nichos que les brindan estos ecosistemas (11).

Dentro de estas aves, el Pisingo (Dendrocygna autumnalis) es de suma importancia, ya que es usado como fuente de proteína de origen animal y es uno de los platos gastronómicos más valorados de la región (12). La ecología y hábitos de vida de $D$. autumnalis se pueden usar para medir el efecto de los metales pesados sobre los ecosistemas en los que habitan. El pisingo es el pato neotropical más abundante, habita en humedales, llanuras inundables y arrozales, se adapta muy bien a los agroecosistemas y se reproduce con facilidad. Debido a que sus hábitos alimenticios se basan en el consumo de granos de maíz y arroz, esta especie se convierte en un bioindicador adecuado de la contaminación con metales pesados. Los hábitos alimenticios y la abundancia de $D$. autumnalis lo convierten en un buen bioindicador de contaminación ambiental $(13,14)$. 
Por lo cual, se estudió la bioacumulación de mercurio y plomo en $D$. autumnalis, perteneciente al ensamble de aves presentes en la región de La Mojana, que está siendo afectada por la minería aurífera y las prácticas agrícolas.

El objetivo de este estudio fue determinar, por primera vez, las concentraciones de $\mathrm{Hg}$ y $\mathrm{Pb}$ en sangre y plumas de $D$. autumnalis (Pisingo) en la región de la Mojana, en virtud de la ausencia de registros de estudios de metales pesados en aves acuáticas de la región.

\section{MATERIALES Y MÉTODOS}

Área de estudio. Esta investigación fue desarrollada en dos estaciones de muestreo localizadas en La Mojana, en la intersección de los cauces de los ríos Magdalena, Cauca y San Jorge, en la depresión Momposina. La primera estación se ubicó en una zona del río San Jorge aledaña a la ciénaga de Ayapel $\left(8^{\circ} 27^{\prime} 34^{\prime \prime} \mathrm{N}\right.$ - 7502'37" W), en el corregimiento de Cecilia (Ayapel-Córdoba), a través de la cual fluye el caño Viloria, subcuenca de la ciénaga de Ayapel, que recibe el influjo de los ríos San Jorge y Cauca. La segunda se sitúo en una zona del río San Jorge contigua a la ciénaga de San Marcos $\left(8^{\circ} 35^{\prime} 19.30^{\prime \prime} \mathrm{N}-75^{\circ} 4^{\prime} 43.40^{\prime \prime} \mathrm{W}\right)$, en la localidad de El Torno (San Marcos-Sucre), la cual es una zona inundable del río San Jorge y colinda con la ciénaga de San Marcos. En el área de estudio se ha documentado contaminación por metales pesados en distintas matrices del ecosistema debido a las cargas contaminadas con metales pesados provenientes de los ríos que circundan a La Mojana. Se ha registrado concentraciones de metales pesados en sedimentos y materia orgánica tales como Cu (48400 ng/g), Zn (79200 ng/g), Ni (58100 ng/g), Pb (3200 $\mathrm{ng} / \mathrm{g}), \mathrm{Cd}(560 \mathrm{ng} / \mathrm{g}), \mathrm{Mn}(41100 \mathrm{ng} / \mathrm{g})$, y Hg (100 ng/g) (5), concentraciones de HgT de $0.097 \pm 0.049 \mu \mathrm{g} / \mathrm{g}$ en sedimentos, $0.191 \pm 0.017$ $\mu \mathrm{g} / \mathrm{g}$ en macrófitas (1), $17.05 \mathrm{ng} / \mathrm{g}$ en arroz (6), en peces como Caquetaia kraussi (150 \pm 57 $\mathrm{ng} / \mathrm{g})$, Hoplias malabaricus (239 $\pm 67 \mathrm{ng} / \mathrm{g})$, Plagioscion surinamensis ( $578 \pm 392 \mathrm{ng} / \mathrm{g}$ ) y Pseudoplatystoma fasciatum $(568 \pm 224 \mathrm{ng} / \mathrm{g})$ (1)(Figura 1).

Colecta de muestras. Los muestreos se realizaron en abril y diciembre de 2019, que corresponden a meses de temporada lluviosa y seca, respectivamente. 10 redes de neblina $(9 \mathrm{~m} \times 2.5 \mathrm{~m})$ se implementaron en la captura de los individuos, las cuales fueron colocadas en sitios considerados de tránsito, alimentación y/o refugio de las aves. El horario de captura estuvo comprendido entre las 7:00 horas y las 18:00 horas. La toma de muestras fue realizada con los permisos competentes otorgados por la Universidad de Córdoba y CORPOMOJANA. Los individuos capturados se identificaron siguiendo las claves taxonómicas de Ayerbe (15). Con la asistencia de un veterinario y dos biólogos, se colectaron entre 1 y $3 \mathrm{ml}$ de sangre de cada ave por medio de la punción de la vena yugular con aguja insulínica. Las muestras fueron almacenadas en tubos con anticoagulante de ácido etilendiaminotetracético (EDTA) y mantenidas en cajas de poliestireno expandido con geles refrigerantes a una temperatura de 4-6 ${ }^{\circ} \mathrm{C}$ para su conservación (16). Se recolectaron alrededor de 10 plumas por cada individuo. Teniendo al ave adecuadamente inmovilizada, se procedió a extraer 4-5 plumas del pecho y 4-5 de otra parte del cuerpo. Las muestras se preservaron en bolsas plásticas herméticamente selladas, se etiquetaron y fueron guardadas para su procesamiento en el laboratorio del grupo de investigación en Aguas, Química Aplicada y Ambiental de la Universidad de Córdoba (16).

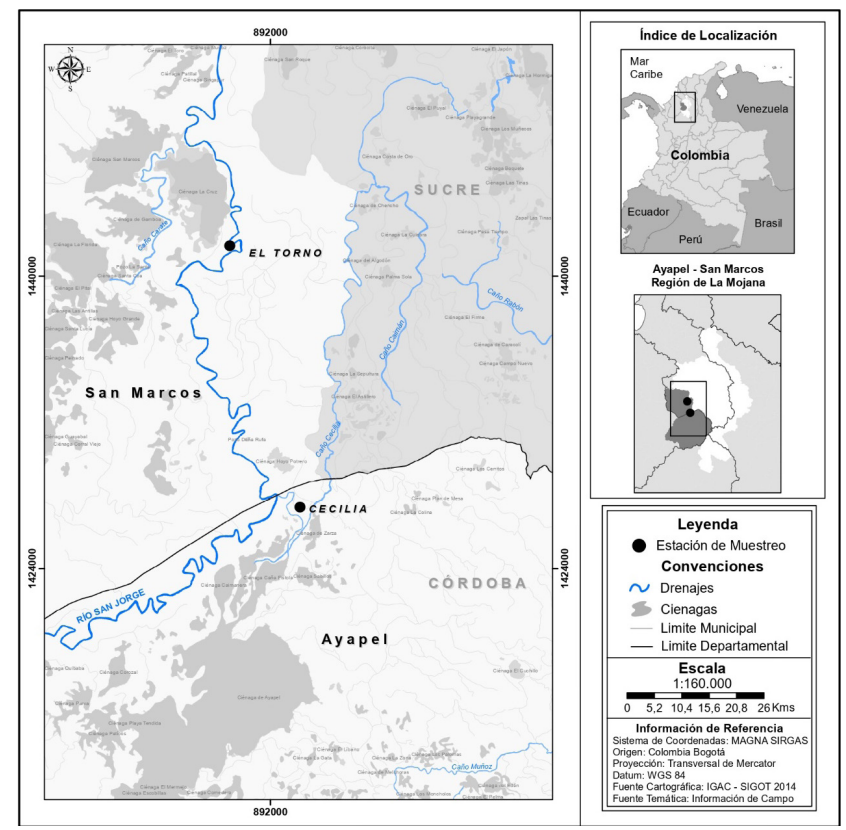

Figura 1. Región de La Mojana (Colombia). Estaciones de muestreo: 1. Caño Viloria-Cecilia. 2. Río San Jorge -El Torno.

Análisis de muestras. La determinación de $\mathrm{Hg}$ y $\mathrm{Pb}$ en sangre de $D$. autumnalis, se realizó mediante espectrometría de absorción atómica con vapor frío (CVAAS) y espectrometría de 
absorción atómica con horno de grafito (GFAAS), respectivamente. Se transfirieron $2 \mathrm{~mL}$ de cada muestra de sangre a un vaso de teflón de 100 $\mathrm{mL}$ de capacidad, al que posteriormente se le agregaron $8 \mathrm{~mL}$ de ácido nítrico concentrado y 2 $\mathrm{mL}$ de peróxido de hidrógeno (30\%). Los vasos se cerraron y su digestión se hizo en un horno microondas a una temperatura de $280^{\circ} \mathrm{C}$, con una presión de 80 bares y una potencia de 1.400 W durante 30 minutos. Para la determinación de $\mathrm{Hg}$, una vez enfriadas, las soluciones se transfirieron a un reactor marcado y el volumen final se ajustó hasta $70 \mathrm{~mL}$ agregando agua desionizada para su posterior análisis (17). Para la determinación de $\mathrm{Pb}$, una vez enfriada, la solución se llevó a un recipiente limpio y seco. Una alícuota de $500 \mu \mathrm{L}$ de la solución sometida a digestión se colocó en un contenedor de muestra y su volumen se ajustó hasta $1 \mathrm{~mL}$ con agua desionizada (17).

La determinación de $\mathrm{Hg}$ y $\mathrm{Pb}$ en plumas de $\mathrm{D}$. autumnalis, se realizó por CVASS y GFAAS, respetivamente. Las muestras de plumas fueron lavadas con agua desionizada y acetona con el fin de la eliminación de metales libres, posteriormente fueron secadas a $60^{\circ} \mathrm{C}$ durante $48 \mathrm{~h}$. Las muestras de plumas fueron digeridas con una mezcla de $\mathrm{H}_{2} \mathrm{SO}_{4}-\mathrm{HNO}_{3}(2: 1, \mathrm{v} / \mathrm{v})$ a $100-110^{\circ} \mathrm{C}$ por $3 \mathrm{~h}, \mathrm{y} \mathrm{KMnO}_{4}(5 \%, \mathrm{p} / \mathrm{v})$ a $100^{\circ} \mathrm{C}$ por $30 \min (18)$.

Análisis estadístico. Para el tratamiento estadístico se determinaron las medias \pm desviación estándar para expresar las concentraciones de mercurio y plomo encontradas en la sangre y las plumas de los 47 individuos capturados. De este modo, se realizó el test de Shapiro-Wilk a partir del cual se corroboró la normalidad $(p<0.05)$ de las distribuciones de las concentraciones de $\mathrm{Hg}$ y $\mathrm{Pb}$ en los tejidos estudiados. Posteriormente se calcularon los rangos de las concentraciones de ambos metales en sangre y plumas para Ayapel y San Marcos con un intervalo de significancia del $95 \%$. Los análisis se realizaron con el paquete estadístico $\mathrm{R}$ versión 3.5.3.

\section{RESULTADOS}

Las concentraciones de $\mathrm{Hg}$ y $\mathrm{Pb}$ en los tejidos de las aves colectadas son presentadas en la Tabla 1, Figura 2 y Figura 3. Se obtuvo en total muestras de 47 aves de la especie $D$. autumnalis,
22 en la primera estación de muestreo (Cecilia) y 25 en la segunda (El Torno). Las concentraciones medias de $\mathrm{Hg}$ en sangre fueron similares en las aves muestreadas en Cecilia y El Torno, $9.87 \pm 10.6 \mathrm{ng} / \mathrm{mL}$ y $5.47 \pm 3.45 \mathrm{ng} / \mathrm{mL}$, siendo levemente superiores en Cecilia. En lo que respecta a la concentración de $\mathrm{Hg}$ en plumas, las concentraciones de la primera estación (475.4 $\pm 180.5 \mathrm{ng} / \mathrm{g}$ ) fueron superiores a los de la segunda (438.46 $\pm 131.25 \mathrm{ng} / \mathrm{g})$. Para $\mathrm{Pb}$ en sangre, la mayor concentración la presentó la primera estación de muestreo (218.5 216.6 $\mathrm{ng} / \mathrm{mL}$ ) mientras que el plomo en plumas fue considerablemente mayor en la segunda estación $(873.59 \pm 412.06)$. Los mayores intervalos de concentración de $\mathrm{Hg}$ y $\mathrm{Pb}$ fueron encontrados en las plumas de los individuos capturados en la primera $(179.2-756.9 \mathrm{ng} / \mathrm{g}$ ) y segunda estación de muestreo (243.99-1643.70 ng/g), respectivamente. Las concentraciones de $\mathrm{Hg}$ y $\mathrm{Pb}$ en plumas fueron superiores a los hallados en sangre.

Tabla 1. Concentración ( $\mathrm{ng} / \mathrm{mL}$ ) de metales pesados ( $\mathrm{Hg} \mathrm{y} \mathrm{Pb}$ ) en sangre y plumas de $D$. autumnalis en la región de La Mojana.

\begin{tabular}{cccccc}
\hline & \multicolumn{5}{c}{ Concentración media \pm SD (Intervalos) } \\
\cline { 2 - 6 } Estación & Sangre $(\mathbf{n g} / \mathbf{m L})$ & \multicolumn{2}{c}{ Plumas (ng/g) } & \\
& $\mathbf{H g}$ & $\mathbf{P b}$ & $\mathbf{H g}$ & $\mathbf{P b}$ & $\mathbf{n}$ \\
\hline \multirow{4}{*}{ E1 } & 9.87 & 218.5 & 475.4 & 5.5 & \\
& \pm 10.6 & \pm 21.6 & \pm 180.5 & \pm 1.2 & \\
& $1.23-$ & $8.73-$ & $179.2-$ & $0.3-$ & \\
& 4.01 & 929.5 & 56.9 & 37 & \\
& 5.47 & 94.11 & 438.46 & 873.59 & \\
E2 & \pm 3.45 & \pm 94.3 & \pm 131.25 & \pm 412.06 & \\
& $1.87-$ & $5.58-$ & $186.37-$ & $243.99-$ & \\
& 14.58 & 280.55 & 676.32 & 1643.70 & \\
\hline Total & \multicolumn{5}{c}{} \\
\hline
\end{tabular}

E1: Estación de muestreo 1. E2: Estación de muestreo 2

Cuatro individuos de $D$. autumnalis, correspondientes a un $8,5 \%$ de los individuos muestreados sobrepasaron el valor de 15 $\mathrm{ng} / \mathrm{mL}$ indicador de intoxicación por $\mathrm{Hg}$ en sangre y posibilidad de muerte, descrito por Scheuhammer et al. (19). La totalidad de las aves presentó concentraciones de $\mathrm{Hg}$ en plumas superiores al indicador de toxicidad de $5.0 \mathrm{ng} / \mathrm{g}$ descrito por Eisler (20). 


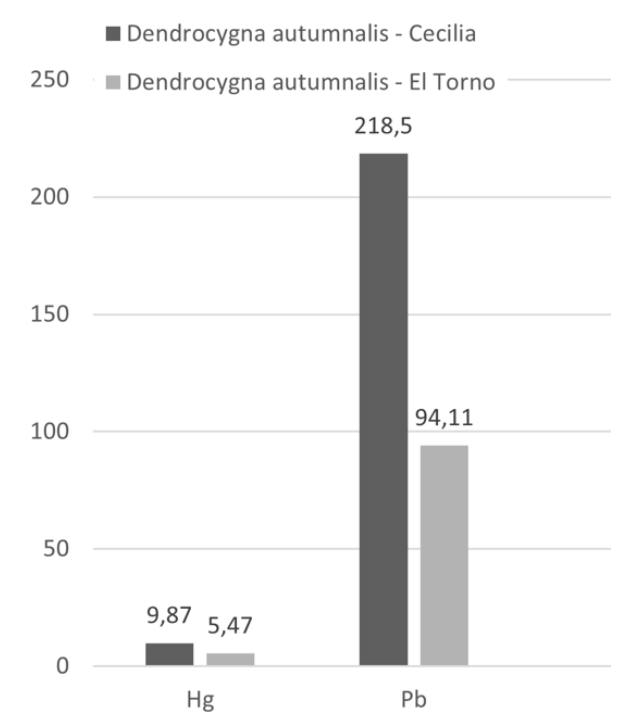

Figura 2. Concentraciones medias de $\mathrm{Hg}$ y $\mathrm{Pb}$ en sangre $(\mathrm{ng} / \mathrm{mL})$ de Dendrocygna autumnalis en Cecilia y El Torno.

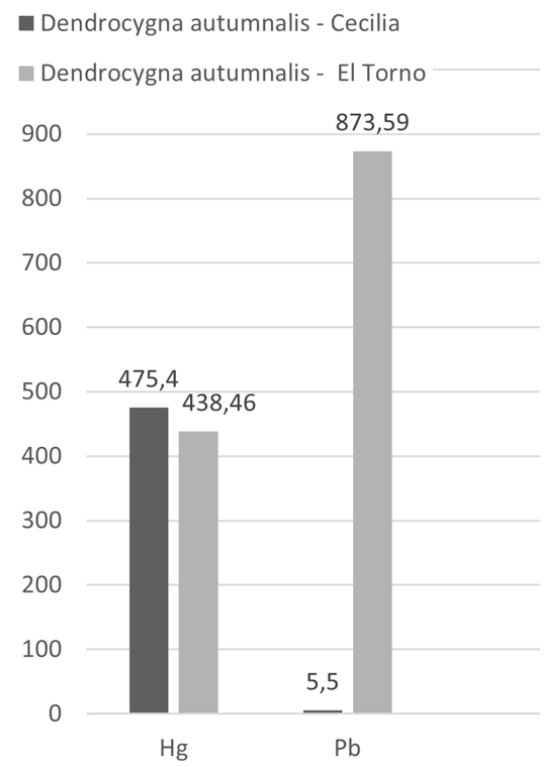

Figura 3. Concentraciones medias de $\mathrm{Hg}$ y $\mathrm{Pb}$ en plumas $(\mathrm{ng} / \mathrm{g})$ de Dendrocygna autumnalis en Cecilia y El Torno.

Respecto a $\mathrm{Pb}$ en sangre, once ejemplares de D. autumnalis, correspondientes al 23,4\% de los individuos muestreados, presentaron intoxicación subclínica (entre 200 y 500 $\mathrm{ng} / \mathrm{mL}$ ), mientras que dos individuos ( $9 \%$ ) manifestaron intoxicación clínica (entre 500 y $1000 \mathrm{ng} / \mathrm{mL}$ ) conforme a lo descrito por Pain (8). La intoxicación subclínica se manifiesta con toxicidad subcelular en el hígado, disminución del tamaño de los huevos, impactos sobre la respuesta inmune, reducción de la tasa de eclosión de huevos, disminución de la motilidad de los espermatozoides y estrés oxidativo, con el riesgo de dañar los componentes de la célula (8). La intoxicación clínica incluye manifestaciones tales como anemia, letargo, desgaste muscular y pérdida de reservas de grasa, diarrea verde, ala caída, pérdida de equilibrio y coordinación y otros signos neurológicos como como parálisis o convulsiones (8). Por otra parte, ninguno de los individuos de $D$. autumnalis sobrepasó el límite de concentración de $\mathrm{Pb}$ en plumas, utilizado como indicador de toxicidad en aves, equivalente a $4000 \mathrm{ng} / \mathrm{g}$, descrito por Tsipoura et al (21).

La totalidad de las plumas muestreadas excedió el límite permisible para $\mathrm{Hg}$ establecido por el Reglamento (UE) 2018/73 (22) de la Unión Europea para carne de pato (de cría y silvestre) que equivale a $40 \mathrm{ng} / \mathrm{g}$. Los 22 individuos de la primera de estación de muestreo registraron una media de concentración de plomo en sangre $(218.5 \pm 216.6 \mathrm{ng} / \mathrm{mL})$ superior al límite permisible de Plomo en carne de pato recomendado por la Unión Europea para niveles máximos para determinados contaminantes en los productos alimenticios (23) de $100 \mathrm{ng} / \mathrm{g}$. De la misma forma los 25 individuos de la segunda estación de muestreo registraron un valor medio de concentración de plomo en plumas $(873.59 \pm 412.06 \mathrm{ng} / \mathrm{g})$ considerablemente superior al límite anteriormente mencionado.

\section{DISCUSIÓN}

En Colombia las evidencias de contaminación por $\mathrm{Hg}$ en aves son escasas. En nuestro estudio, las mayores concentraciones de $\mathrm{Hg}$ (475.4 180.5 $\mathrm{ng} / \mathrm{g}$ ) fueron inferiores a las concentraciones registradas en otros estudios de contaminación por metales pesados en aves de Colombia. SierraMárquez et al (24) reportaron la presencia de este metal en plumas $(840 \pm 50 \mathrm{ng} / \mathrm{g})$ de aves insectívoras, nectívoras y frugívoras en el parque natural Las orquídeas, Antioquia. Burgos-Nuñez et al (25) determinaron en la Bahía de Cispatá - Córdoba, cantidades de $\mathrm{Hg}$ en plumas de Fregata magnificens de $10190 \mathrm{ng} / \mathrm{g}$, las cuales son superiores a todas las concentraciones de $\mathrm{Hg}$ halladas en este estudio. Por otra parte, las mayores cantidades de mercurio cuantificadas en sangre de Dendrocygna autumnalis (9.87 \pm 10.6 $\mathrm{ng} / \mathrm{mL}$ ) fueron inferiores a las obtenidas por Albuja et al (26) en 54 especies de aves silvestres asociadas a tres regiones mineras en Ecuador, con un valor máximo de $162.16 \mathrm{ng} / \mathrm{mL}$. Las mayores concentraciones medias de $\mathrm{Pb}$ en sangre 
de Dendrocygna autumnalis (218.5 $\pm 216.6 \mathrm{ng} /$ $\mathrm{mL}$ ) fueron superiores a las encontradas en Anas platyrhynchos ( $112 \pm 66 \mathrm{ng} / \mathrm{mL})$ y menores que las halladas en Aythya ferina ( $926 \pm 463 \mathrm{ng} / \mathrm{mL}$ ) durante una investigación en aves acuáticas (27) en España. Las mayores mediciones de $\mathrm{Pb}$ en plumas de $D$. autumnalis $(873.59 \pm 412.06 \mathrm{ng} / \mathrm{g}$ ) fueron inferiores a las obtenidas por Garitano et al. (28) en un estudio realizado en especies de la familia Tinamidae asociadas a zonas mineras en Bolivia, en las que se midieron concentraciones medias de este metal correspondientes a 7764.48 $\mathrm{ng} / \mathrm{g}$ en este tejido. En un estudio realizado por Ferreyra et al. (29), cuyo objetivo fue determinar la incidencia de la ingestión de perdigones de plomo usados en la cacería y consumidos por varias especies del género Dendrocygna, se determinaron concentraciones máximas de este metal de $17100 \pm 5330 \mathrm{ng} / \mathrm{g}$, mayores a las registradas en nuestra investigación. Los anteriores estudios muestran cómo las actividades antrópicas son responsables de la liberación de metales pesados al medio, los cuales terminan en los tejidos de las aves. Dichos metales, al entrar en contacto con el agua modifican sus parámetros fisicoquímicos (30).

Factores como las condiciones fisicoquímicas del agua y los sedimentos también juegan un rol crucial en la dinámica contaminante de estos metales, dado que favorecen su proceso bioacumulativo e incrementan las concentraciones de los mismos en los ecosistemas acuáticos y los organismos, a través de la biomagnificación e incorporación en la red trófica (5). Estos procesos dependen en gran medida de los flujos de agua y el desplazamiento del material particulado, así como de su circulación en los organismos que componen la red trófica, a través de la biomagnificación desde las macrófitas hasta los eslabones tróficos superiores de la biota asociada a los ecosistemas acuáticos de la región de La Mojana (1). Todo esto influye directamente en la dinámica biológica de los organismos que conforman la red trófica a la que pertenece $D$. autumnalis.

Las cantidades de metales pesados halladas en $D$. autumnalis, pueden explicarse debido a aspectos puntales en la ecología de la especie. En este sentido, los hábitos alimenticios de los individuos juegan un rol crucial en los procesos de bioacumulación y biomagnificación de contaminantes como los metales pesados (31). D. autumnalis es una especie de pato con hábitos alimenticios omnívoros, cuya dieta se basa mayoritariamente en granos de plantas y pequeños invertebrados (13). Los humedales constituyen el principal nicho para las aves forrajeras, como D. autumnalis, debido a que son ecosistemas altamente productivos, ofertando una gran cantidad de semillas e invertebrados acuáticos a dichas especies. Sin embargo, los humedales pueden ser muy propicios para la producción y bioacumulación de $\mathrm{MeHg}$, ya que el crecimiento de los cultivos en aguas limita la radiación solar sobre el humedal generando condiciones biofísicas adecuadas para permitir una poca degradación y mayor trasporte de mercurio en los cultivos, en específico el arroz (32). Los grados de contaminación por $\mathrm{Hg}$ registradas en esta investigación, se deben a que $D$. autumnalis se alimenta de cultivos de arroz contaminados con este metal. En trabajos investigativos desarrollados en el área de estudio $(6,33)$ se determinó contaminación por dicho metal en cultivos de arroz de La Mojana, con concentraciones de $17.05 \mathrm{ng} / \mathrm{g}$ y concentraciones de $0.021 \mu \mathrm{g} / \mathrm{g}$, respectivamente. Los sedimentos, el agua y el consumo de macroinvertebrados también representan fuentes de contaminación para esta especie. Marrugo et al (1) registraron $0.097 \pm 0.049 \mu \mathrm{g} / \mathrm{g}$ de $\mathrm{HgT}$ en sedimentos y $0.191 \pm 0.017 \mu \mathrm{g} / \mathrm{g}$ en macrófitas de la Mojana.

El mercurio tiende a acumularse en el manto de sedimentos de los humedales y a ser transportado a través de la columna de agua. La especie $D$. autumnalis al estar continuamente en estas matrices ambientales recibe dosis de mercurio por contacto directo con la piel y a través de la ingesta de agua. Además, los macroinvertebrados de los que se alimenta tienen como fuente alimenticia plantas macrófitas y sedimentos. El proceso de biomagnificación del $\mathrm{Hg}$ inicia en los sedimentos, pasando a las macrófitas, y de estas a los macroinvertebrados acuáticos de los que se alimenta $D$. autumnalis (34). Las concentraciones de $\mathrm{Pb}$ registradas en sangre y plumas de $D$. autumnalis se pueden explicar por la acumulación de $\mathrm{Pb}$ en matrices ambientales que sirven como sustrato alimenticio para esta especie como los cultivos de arroz, los cuales requieren altas concentraciones de agroquímicos, que son ricos en metales pesados (35). Estos contaminantes se fijan en el arroz y se diseminan en otros estratos productores de la red alimentaria (5). Otra posible razón que explique la contaminación con $\mathrm{Pb}$ se halla en el hecho de que este metal es utilizado como munición para la caza de $D$. autumnalis, propiciando que los individuos de estas poblaciones ingieran munición dispersa en el ambiente para ser utilizada como gastrolitos, es decir, piedras que facilitan la digestión (29). 
La especie $D$. autumnalis presentó bajos niveles de toxicidad para $\mathrm{Hg}$ en sangre, sin embargo, el total de los individuos muestreados de esta especie superó el límite de toxicidad de $5 \mathrm{ng} / \mathrm{g}$ para $\mathrm{Hg}$ en plumas descrito por Eisler (20). Una proporción de los individuos de $D$. autumnalis presentó concentraciones de $\mathrm{Pb}$ en sangre que indican algún grado de toxicidad de acuerdo con lo descrito por Pain et al (8). Los anteriores niveles de toxicidad son un claro indicio de que esta especie puede estar viéndose afectada por los afectos adversos que genera el $\mathrm{Hg}$ y el $\mathrm{Pb}$ en los sistemas hematopoyético, vascular, nervioso, renal, inmune y reproductivo de las aves $(7,8)$. Derivando en la alteración de rasgos comportamentales que son vitales para la supervivencia de la especie $(7,8)$.

La alta biodiversidad de la región de La Mojana posibilita el uso de la fauna en la alimentación de las poblaciones humanas asentadas en ella. Es común la caza y el aprovechamiento de especies de reptiles tales como: Trachemys callirostris, Iguana iguana, Caiman crocodilus fuscus, Podocnemis lewyana y aves como: Anas discors, Dendrocygna viduata, Dendrocygna autumnalis (12). La caza de Pisingo se realiza con el propósito de usarla para consumo como base de proteína de origen animal y hace parte de la gastronomía de la región (12). La ingesta de carne de Pisingo con presencia de metales pesados genera en los animales alteraciones comportamentales, fisiológicas, reproductivas, daño oxidativo al ADN, reducción de las tasas de crecimiento y del número de huevos (21). En los humanos el mercurio afecta el sistema nervioso y el plomo causa déficit en el coeficiente intelectual, trastornos del comportamiento, así como con una disminución del volumen cerebral en adultos (36). Diversos hallazgos registran contaminación con $\mathrm{Hg}$ en humanos provenientes del consumo de otras especies asociadas a los humedales de La Mojana (17). De forma que el consumo de carne de pisingo contaminada por $\mathrm{Hg}$ se suma al consumo de peces, arroz e hicoteas también contaminadas, poniendo en riesgo la salud de los habitantes de la zona $(1,6,33)$. En un estudio realizado en La Mojana, personas con presencia de mercurio en su organismo manifestaron síntomas tales como dolor de cabeza, falta de energía, agotamiento, nerviosismo o irritabilidad y preocupación en exceso (37). El mercurio y sus formas metiladas generan toxicidad desde muy bajas concentraciones, afectando en mayor medida al sistema nervioso central, sistema renal y hemático (19). La exposición al $\mathrm{Pb}$ en humanos afecta la salud, especialmente en niños pequeños y mujeres en estado de gestación (38). La exposición de fetos y niños al Pb está relacionada con trastornos neurológicos como el trastorno por déficit de atención e hiperactividad y déficits cognitivos (39). El plomo puede generar trastornos durante la gestión, el parto y en los neonatos (40). Por lo tanto, los consumidores pueden incurrir en riesgos para la salud por el consumo prolongado de carne de Pisingo contaminada con $\mathrm{Hg}$ y $\mathrm{Pb}$.

En conclusión, la especie $D$. autumnalis presenta niveles de contaminación por $\mathrm{Hg}$ y $\mathrm{Pb}$ preocupantes que revelan un gran impacto de las actividades antrópicas, como la minería y las actividades agrícolas, en la ecorregión de La Mojana. Para conocer de forma más precisa los procesos contaminantes generados por metales pesados en la avifauna de la región se hacen necesarios estudios en mayor diversidad de especies, en mayor cantidad de sitios de muestreo y en periodos de tiempo más largos. Se recomienda que se realicen estudios midiendo metales pesados en carne de pato ya capturado para consumo, y a partir de los datos suministrados por esta investigación, las autoridades ambientales competentes diseñen y ejecuten estrategias para la evaluación y conservación de los ecosistemas acuáticos y la avifauna asociada a estos en La Mojana

La ingesta de carne de pisingo contaminada representa un problema de salud pública ya que los metales pesados se bioacumulan en estos organismos luego de pasar por varios niveles de la red trófica. Razón por la cual es necesario informar y educar a las poblaciones de La Mojana sobre el consumo de carne de pisingo, a fin de proteger a los individuos en edad gestacional, niños y población general de los efectos del mercurio y plomo en sus organismos.

\section{Conflicto de interés}

No existen conflictos de interés.

\section{Agradecimientos}

Los autores agradecen a la Universidad de Córdoba, Montería-Colombia, al Grupo de Química del Agua Aplicada y Ambiental y al Laboratorio de Toxicología y Gestión Ambiental. 


\section{REFERENCIAS}

1. Marrugo Negrete J, Pinedo-Hernández J, Paternina-Uribe R, Quiroz-Aguas L, PachecoFlorez S. Distribución espacial y evaluación de la contaminación ambiental por mercurio en la región de la Mojana, Colombia. Rev MVZ Cordoba. 2018; 23(S):7062-7075. https://doi.org/10.21897/rmvz.1481

3. Carlos F, Lina MES, Sergio R, César A, Marcela $Q$, Óscar $A$, et al. Identificación espacial de los sistemas de humedales continentales de Colombia. Biota Colomb. 2016; 16(3):4462. https://doi.org/10.21068/c2016s01a03

3. Ramsar C. The List of Wetlands of International Importance; RAMSAR Secretariat: Gland, Switzerland, 2021.

4. Marrugo J, Lans E, Benítez L. Hallazgo de mercurio en peces de la Ciénaga de Ayapel, Córdoba, Colombia. Rev MVZ Córdoba. 2007; 12(1): 878-886 https://doi. org/10.21897/rmvz.432

5. Marrugo-Negrete J, Pinedo-Hernández J, Combatt EM, Bravo AG, Díez S. Floodinduced metal contamination in the topsoil of floodplain agricultural soils: A casestudy in Colombia. Land Degrad Dev. 2019; 30(17):2139-2149. https://doi. org/10.1002/ldr.3398

6. Argumedo MP, Vergara C, Vidal J, Marrugo JL. Evaluación de la concentración de mercurio en arroz (Oryza sativa) crudo y cocido procedente del municipio de San Marcos- Sucre y zona aurífera del municipio de Ayapel - Córdoba. Rev Univ Ind Santander Salud. 2015; 47(2):169177. https://revistas.uis.edu.co/index.php/ revistasaluduis/article/view/4826/4964

7. Whitney MC, Cristol DA. Impacts of sublethal mercury exposure on birds: A detailed review. Rev Environ Contam Toxicol. 2018; 244:113-163. https://doi. org/10.1007/398 2017 4

8. Pain DJ, Mateo R, Green RE. Effects of lead from ammunition on birds and other wildlife: A review and update. Ambio. 2019; 48(9):935-953. http://dx.doi.org/10.1007/ s13280-019-01159-0
9. Ruiz-Guerra C. Lista de Aves Acuáticas de Colombia. Asociación Calidris; 2012. http:// dx.doi.org/10.13140/RG.2.1.2511.8244

10. Zhang WW, Ma JZ. Waterbirds as bioindicators of wetland heavy metal pollution. Procedia Environ Sci. 2011; 10:2769-2774. http:// dx.doi.org/10.1016/j.proenv.2011.09.429

11. Linares AJ, Carrillo FM, González CM, Vergara DL, Ortega LÁ, Ruiz VR, et al. Caracterización en la dinámica espacial de los macrohábitats acuáticos en la región de La Mojana. Instituto de Investigación de Recursos Biológicos Alexander von Humboldty: Universidad de Córdoba; 2018. http://repository.humboldt.org.co/ handle/20.500.11761/34994

12. Valencia-Parra E, de La Ossa VJ. Patrones de uso de fauna silvestre en el bajo río San Jorge, Sucre, Colombia. Rev Colomb Cienc Anim RECIA. 2016; 8(S1):276. http:// dx.doi.org/10.24188/recia.v8.n0.2016.382

13. Ramirez $E$, Arguello $M$, Ilizaliturri $C$, Tintos $A$, Mejia J, Borja I. A brief review of the use of biomarkers in Mexico s aquatic ecosystems pollution assessment: 2001 2002. Lat Am J Aquat Res. 2018; 46(5):860-879. http:// dx.doi.org/10.3856/vol46-issue5fulltext-1

14. Osten JR, Soares AMVM, Guilhermino L. Black-bellied whistling duck (Dendrocygna autumnalis) brain cholinesterase characterization and diagnosis of anticholinesterase pesticide exposure in wild populations from Mexico. Environ Toxicol Chem. 2005; 24(2):313-317. https://doi. org/10.1897/03-646.1

15. Ayerbe Quiñonez F. Guía ilustrada de la Avifauna colombiana. $1^{a}$ ed. Bogotá, Colombia: Wildlife Conservation Society; 2018. 
16. Muñoz-García C, Rendón-Franco E, LópezDíaz O, Ruiz RR, Arechiga-Ceballos N, Villanueva $C$, et al. Colecta y conservación de muestras de fauna silvestre en condiciones de campo. UAM, Unidad Xochimilco: México; 2016. https://casadelibrosabiertos.uam.mx/ gpd-colecta-y-conservacion-de-muestrasde-fauna-silvestre-en-condiciones-decampo.html

17. Calao CR, Marrugo JL. Efectos genotóxicos en población humana asociados a metales pesados en la región de La Mojana, Colombia, 2013. Biomedica. 2015; 35(Supl.2):139151. http://dx.doi.org/10.7705/biomedica. v35i0.2392

18. Burgos N S, Marrugo N J, Navarro F A, Urango C I. Mercury in Pelecanus occidentalis of the Cispata bay, Colombia. Rev MVZ Cordoba. 2014; 19(2):4168-4174. https://doi. org/10.21897/rmvz.110

19. Scheuhammer AM, Meyer MW, Sandheinrich MB, Murray MW. Effects of environmental methylmercury on the health of wild birds, mammals, and fish. Ambio. 2007; 36(1):12-18. http://dx.doi.org/10.1579/0 0447447(2007)36[12:eoemot]2.0.c0;2

20. Eisler R. Mercury hazards to living organisms. Boca Ratón, FL, Estados Unidos de América: CRC Press; 2006. http://dx.doi. org/10.1201/9781420008838

21. Tsipoura N, Burger J, Newhouse M, Jeitner C, Gochfeld M, Mizrahi D. Lead, mercury, cadmium, chromium, and arsenic levels in eggs, feathers, and tissues of Canada geese of the New Jersey Meadowlands. Environ Res. 2011; 111(6):775-784. http://dx.doi. org/10.1016/j.envres.2011.05.013

22. Commission Regulation (EU) $2018 / 73$ of 16 January 2018 amending Annexes II and III to Regulation (EC) No 396/2005 of the European Parliament and of the Council as regards maximum residue levels for mercury compounds in or on certain products Text with EEA. Off J. Eur. Union; 2018. http:// data.europa.eu/eli/reg/2018/73/oj

23. Commission Regulation. No $1881 / 2006$ of 19 December 2006 setting maximum levels for certain contaminants in foodstuffs. Official Journal of European Union; 2006. http:// data.europa.eu/eli/reg/2006/1881/oj
24. Sierra-Marquez L, Peñuela-Gomez S, FrancoEspinosa L, Gomez-Ruiz D, Diaz-Nieto J, Sierra-Marquez J, et al. Mercury levels in birds and small rodents from Las Orquideas National Natural Park, Colombia. Environ Sci Pollut Res Int. 2018; 25(35):35055-35063. http://dx.doi.org/10.1007/s11356-0183359-2

25. Burgos-Núñez $S$, Navarro-Frómeta $A$, Marrugo-Negrete J, Enamorado-Montes G, Urango-Cárdenas I. Polycyclic aromatic hydrocarbons and heavy metals in the Cispata Bay, Colombia: A marine tropical ecosystem. Mar Pollut Bull. 2017; 120(12):379-386. http://dx.doi.org/10.1016/j. marpolbul.2017.05.016

26. Albuja L, Montalvo D, Cáceres F, Jácome $\mathrm{N}$. Niveles de mercurio en aves silvestres de tres regiones mineras del sur del Ecuador. Politécnica. 2012; 30(3):18. http:// bibdigital.epn.edu.ec/handle/15000/5058

27. Martinez-Haro M, Green AJ, Mateo R. Effects of lead exposure on oxidative stress biomarkers and plasma biochemistry in waterbirds in the field. Environ Res. 2011; 111(4):530-538. https://doi.org/10.1016/j. envres.2011.02.012

28. Garitano-Zavala A, Cotín J, Borràs M, Nadal J. Trace metal concentrations in tissues of two tinamou species in mining areas of Bolivia and their potential as environmental sentinels. Environ Monit Assess. 2010; 168(1-4):629-644. http:// dx.doi.org/10.1007/s10661-009-1139-7

29. Ferreyra $H$, Romano $M$, Beldomenico $P$, Caselli A, Correa A, Uhart $M$. Lead gunshot pellet ingestion and tissue lead levels in wild ducks from Argentine hunting hotspots. Ecotoxicol Environ Saf. 2014; 103:74-81. http://dx.doi.org/10.1016/j. ecoenv.2013.10.015

30. Flores CM, Del Angel E, Frías DM, Gómez AL. Evaluación de parámetros fisicoquímicos y metales pesados en agua y sedimento superficial de la Laguna de las Ilusiones, Tabasco, México. Tecnol cienc agua. 2018; 9(2):39-57. http://dx.doi.org/10.24850/jtyca-2018-02-02 
31. Francis EA. Paramount roles of wild birds as bioindicators of contamination. Int Int J Avian Wildl Biol. 2017; 2(6). http://dx.doi. org/10.15406/ijawb.2017.02.00041

32. Windham-Myers $L$, Fleck JA, Ackerman JT, Marvin-DiPasquale $M$, Stricker CA, Heim WA, et al. Mercury cycling in agricultural and managed wetlands: a synthesis of methylmercury production, hydrologic export, and bioaccumulation from an integrated field study. Sci Total Environ. 2014; 484:221-231. http://dx.doi. org/10.1016/j.scitotenv.2014.01.033

33. Argumedo García MP, Consuegra-Solórzano A, Vidal-Durango JV, Marrugo-Negrete JL. Exposición a mercurio en habitantes del municipio de San Marcos (Departamento de Sucre) debida a la ingesta de arroz (Oryza sativa) contaminado. Rev Salud Pública. 2013; 15(6):903-915. https://revistas.unal. edu.co/index.php/revsaludpublica/article/ view/40760

34. Asante $\mathrm{CK}$, Hobson $\mathrm{KA}$, Bond AL, Jardine TD. Resource partitioning among five species of waterfowl (Anasspp.) at an autumn migratory stopover: combining stable isotope and mercury biomarkers. Can J Zool. 2017; 95(4):279-286. http://dx.doi. org/10.1139/cjz-2016-0063

35. Martí L, Filippini MF, Bermejillo A, Troilo S, Salcedo C, Valdés A. Monitoreo de cadmio y plomo en los principales fungicidas cúpricos comercializados en Mendoza, Argentina. Rev Fac Cienc Agrar. 2009; 41(2):109-116. https://bdigital.uncu.edu.ar/3185
36. Aendo $\mathrm{P}$, Netvichian $\mathrm{R}$, Khaodhiar $\mathrm{S}$, Thongyuan S, Songserm T, Tulayakul P. Pb, $\mathrm{Cd}$, and $\mathrm{Cu}$ play a major role in health risk from contamination in duck meat and offal for food production in Thailand. Biol Trace Elem Res. 2020; 198(1):243-252. https:// doi.org/10.1007/s12011-020-02040-y

37. Gracia HL, Marrugo NJL, Alvis REM. Contaminación por mercurio en humanos y peces en el municipio de Ayapel, Córdoba, Colombia, 2009. Rev Fac Nac Salud Pública. 2010; 28(2):118-124 https://revistas.udea. edu.co/index.php/fnsp/article/view/1753

38. Spanier AJ, McLaine P, Gilden RC. Screening for elevated blood lead levels in children and pregnant women. JAMA. 2019; 321(15):1464-1465. https://doi. org/10.1001/jama.2019.2594

39. Téllez-Rojo MM, Bautista-Arredondo LF, Richardson V, Estrada-Sánchez D, ÁvilaJiménez $L$, Ríos $C$, et al. Intoxicación por plomo y nivel de marginación en recién nacidos de Morelos, México. Salud Publica Mex. 2017; 59(3):218-226. https:// saludpublica.mx/index.php/spm/article/ view/8045

40. Jameil NA. Maternal serum lead levels and risk of preeclampsia in pregnant women: a cohort study in a maternity hospital, Riyadh, Saudi Arabia. Int J Clin Exp Pathol. 2014; 7(6):3182-3189. https://www.ncbi.nlm. nih.gov/pubmed/25031738 